\title{
Improving Sample Preparation Methods to Assess Nanoparticle Agglomeration using TEM
}

Jiwen Zheng

Food and Drug Administration, Center for Devices and Radiological Health, Office of Science and Engineering Laboratories, Division of Chemistry and Materials Science, Silver Spring, MD 20993

Many nanoparticles agglomerate when exposed to biological media or blood, affecting cellular uptake and biodistribution [1,2]. The comprehensive physicochemical characterization (such as size, agglomeration, etc.) of both primary nanoparticles (nanoparticles as produced), and nanoparticles under conditions relevant to in vitro or in vivo studies is critical for accurate toxicity evaluation of nanoparticles.

The techniques to assess nanoparticle size and agglomeration include spectroscopy (e.g. ultraviolet-visible spectroscopy (UV-Vis)), light scattering (e.g. dynamic light scattering (DLS)) and electron microscopy (e.g. transmission electron microscopy (TEM)). Among these techniques, TEM offers great benefits in evaluating nanoparticle size and size distribution due to its direct visualization nature and ultrahigh resolution. However, the drawback associated with TEM sample preparation prevents it from evaluating the agglomeration of nanoparticles. A commonly used technique to prepare nanoparticles for TEM imaging is drop casting method where an aliquot of nanoparticle solution is being dropped on TEM grid, followed by blotting and air-drying. This method, very often, suffers from the formation of unexpected agglomeration of nanoparticles on the TEM grid due to surface tension during air-drying step.

This problem can be resolved by vitrifying (cryo-freezing) or electrospraying nanoparticles on the TEM grid. We tested a wide variety of nanoparticles (gold, silver and iron oxide) and the results showed unexpected agglomeration of nanoparticles has been significantly reduced. We expect that these methods can be routinely applied for the evaluation of size and agglomeration of nanoparticles, along with other spectroscopic and light scattering techniques.

\section{References:}

[1] Zook JM, Maccuspie RI, Locascio LE, Halter MD, Elliott JT. Nanotoxicology 5 (2011), p. 517.

[2] Keene AM, Peters D, Rouse R, Stewart S, Rosen ET, Tyner KM. Nanomedicine 7 (2012), p. 199. 
